\title{
Tunable Platform Tolerant Antenna Design for RFID and IoT Applications Using Characteristic Mode Analysis
}

\author{
Abubakar Sharif $\left(\mathbb{D},{ }^{1}\right.$ Jun Ouyang $\left(\mathbb{0},{ }^{1}\right.$ Feng Yang, \\ Rui Long, ${ }^{1}$ and Muhammad Kamran Ishfaq ${ }^{2}$ \\ ${ }^{1}$ School of Electronic Science and Engineering, University of Electronic Science and Technology of China (UESTC), Chengdu, China \\ ${ }^{2}$ Wireless Communication Centre, Universiti Teknologi Malaysia, Johor Bahru, Malaysia \\ Correspondence should be addressed to Jun Ouyang; yjou@uestc.edu.cn
}

Received 2 February 2018; Accepted 4 March 2018; Published 10 May 2018

Academic Editor: Masood Ur-Rahman

Copyright (C) 2018 Abubakar Sharif et al. This is an open access article distributed under the Creative Commons Attribution License, which permits unrestricted use, distribution, and reproduction in any medium, provided the original work is properly cited.

Radio frequency identification (RFID) is a key technology to realize IoT (Internet of Things) dreams. RFID technology has been emerging in sensing, identification, tracking, and localization of goods. In order to tag a huge number of things, it is cost-effective to use one RFID antenna for tagging different things. Therefore, in this paper a platform tolerant RFID tag antenna with tunable capability is proposed. The proposed tag antenna is designed and optimized using characteristic mode analysis (CMA). Moreover, this tag antenna consists of a folded patch wrapped around FR 4 substrate and a feeding loop element printed on a paper substrate. The inductive feeding loop is stacked over folded patch and it provides impedance match with RFID chip. Because of separate radiating and feeding element, this tag antenna has a versatility of impedance matching with any RFID chip. Furthermore, this tag is able to cover American RFID band (902-928 MHz) and can be tuned to European RFID band ( $865-868 \mathrm{MHz}$ ) by adding tunable strips. In order to demonstrate platform tolerant operation, the read range of RFID tag is measured by mounting it on different materials. The maximum read range of RFID tag is $4.5 \mathrm{~m}$ in free space or on dielectrics and $6.5 \mathrm{~m}$ above $200 \times 200 \mathrm{~mm}^{2}$ metal plate, respectively.

\section{Introduction}

The modern wireless technologies are driving this world towards a new paradigm which includes Internet of Things (IoT) and Internet of Everything (IoE). In IoT world, everything will have its own unique identity. The Internet of Things (IoT) will provide an emerging integrated wireless platform, where physical and virtual things can be uniquely identified on a global scale and are connected with a global network. IoT becomes prevalent in both research and industries due to its unique emerging applications like smart cities $[1,2]$, connected vehicles [2], healthcare monitoring [3-5], and so on [2]. Radio frequency identification (RFID) is one of the vital technologies of IoT since it enables a device (object) not only to share its unique digital code across a network wirelessly, but also to capture its physical status for localization on a global scale [6]. RFID in combination with IoT has opened a new paradigm and new era of applications [7]. A descriptive modal to enable an IoT network integrated with RFID technology is shown in Figure 1.

The thing's basic information will be stored in electronic RFID tag in form of electronic product code (EPC) or universal identification (UID) and this information can be read by wireless RFID reader, which can be accessed from anywhere through Internet [8-10].

Passive ultrahigh (UHF) RFID tags are more attractive for most of applications because of their long-read range and high data rate capabilities. Moreover, the low cost, easily printable structure of UHF RFID tags enables them to integrate or embed inside tagged objects, to give them a unique attribute. However, UHF RFID tags are much more sensitive towards host tagging objects such as metals, wood, and glass. This sensitivity, on one side, is good for exploiting UHF RFID tags as sensing device. On the other hand, this sensitivity towards different surfaces requires a separate UHF RFID 


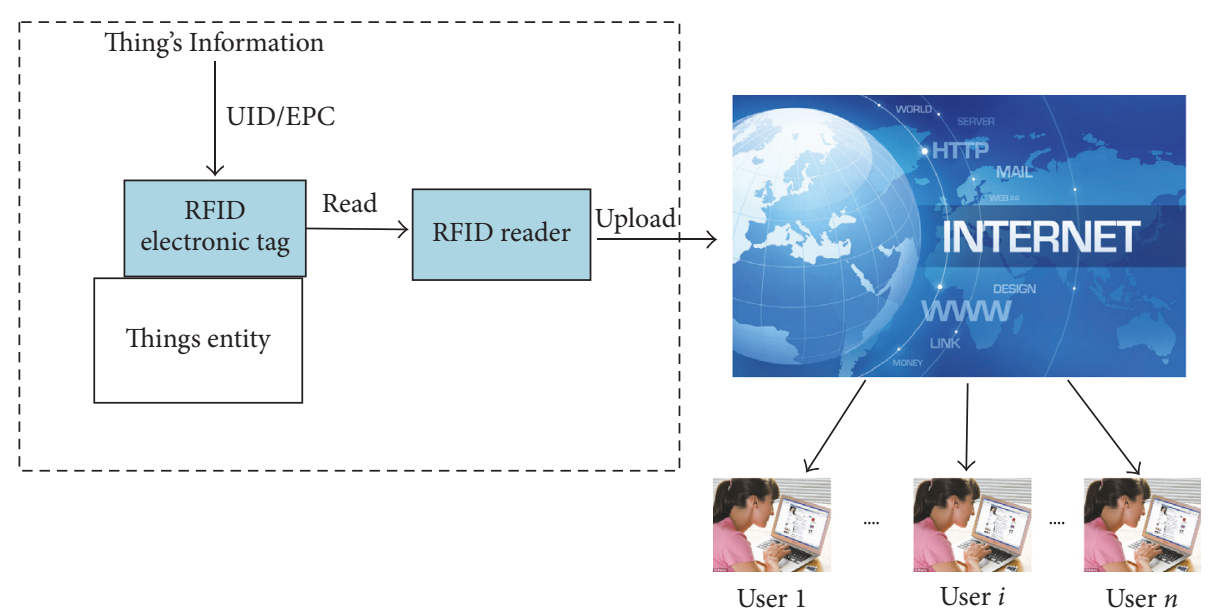

FIgURE 1: IoT descriptive modal integrated with RFID.

tag design for each tagged entity $[7,11]$. Therefore, it is advantageous and cost-effective to design one low cost, platform tolerant antenna solution to tag most of the things.

In literature, there are many solutions available to solve this problem of platform tolerant UHF RFID tag especially for counter metallic surface effects [12,13]. In [14], a dual layer PIFA antenna was introduced for platform tolerant applications. However, the performance of this design is highly depending on location of vias. Also, the dual layer structure with introduction of vias makes it a costly solution. A single layer coupled loop based antenna fabricated on $3 \mathrm{~mm}$ thick FR 4 substrate was proposed in [15], for tagging metal and low permittivity dielectric materials. Although this proposed antenna works on metal with read range of $6 \mathrm{~m}$, its major drawback is substrate thickness. In [16], a stepped impedance coupled patch antenna was designed on a thin substrate. However, this coupled patch has small bandwidth especially on metallic surfaces. Another folded patch antenna with digital edge serration was designed in [17], for tagging metallic objects. This antenna was wrapped around $3 \mathrm{~mm}$ thick substrate with RFID chip mounted on vertical edge, which leads to a structure which is expensive and difficult to fabricate. Also, the vertical chip mounting configuration is more vulnerable to damage. Another solution was proposed in [18] by employing a hybrid dual port tag antenna, which consists of a dipole embedded with a patch antenna. This design has large size with $3.2 \mathrm{~mm}$ substrate thickness.

There are many techniques to design an efficient platform tolerant antenna such as impedance analysis, employing metamaterials (electromagnetic bandgap (EBG), artificial magnetic conductance (AMC)), and circuit analysis.

Characteristic mode analysis (CMA) in combination with theory of characteristic modes (TCM) is becoming a prevalent systematic tool to design efficient terminal antennas. The use of TCM in antenna design was not new topic. It was first proposed by Garbacz and later redefined by Harrington and Mautz [19]. However, in some recent years, TCM was revisited to get physical insight into antenna designing. TCM is more attractive and systematic approach because of its following features $[19,20]$. (1) It provides a more systematic design approach rather than a brute-force. (2) It also allows designer to get physical insight into antenna's operation. (3) It can help to find resonance frequency of specific mode. (4) It also allows finding the optimum feed arrangement to excite these specific modes. Moreover, there are very few designs available using CMA for RFID [21, 22] and IoT applications [23].

In this paper, a tunable platform tolerant folded patch antenna is proposed for UHF RFID and IoT applications using characteristic mode analysis. This antenna consists of a folded patch (wrapped around FR 4 substrate) and a small inductive feeding loop. The feeding loop printed on a paper substrate is stacked over folded patch configuration. The proposed folded patch is optimized using CMA to resonate at $915 \mathrm{MHz}$. The small feeding loop is connected with RFID chip to provide a conjugate impedance match.

Because of separate radiating and feeding element, the proposed tag antenna has a versatility of impedance matching with any RFID chip. Furthermore, this tag is able to cover US RFID band and can be tuned to European RFID band by adding tunable strips. In order to validate platform tolerant operation, the read range of RFID tag is measured by mounting it on different materials. The maximum read range of RFID tag is $4.5 \mathrm{~m}$ in free space or on dielectrics and $6.5 \mathrm{~m}$ above $200 \times 200 \mathrm{~mm}^{2}$ metal plate, respectively. Moreover, this tag can also be equipped with IoT based sensors to sense environmental effects and processes, thanks to its platform tolerant capability. The low cost, tunable, and platform tolerant feature of this antenna makes it suitable for RFID and IoT applications.

\section{Characteristic Mode Analysis}

2.1. Theory of Characteristic Modes (TCM). Characteristic modes can be defined as orthogonal surface current modes of an arbitrary shaped conducting body, which totally depend on its shape and size and are independent of any feed source.

Moreover, characteristic modes (CM) can be derived by solving eigenvalue equations, obtained from Method of Moments (MoM) based impedance matrix as follows: 


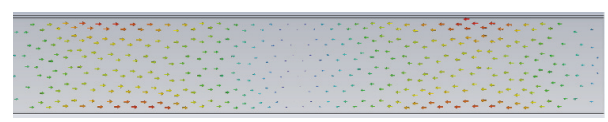

Mode $1\left(J_{1}\right)$

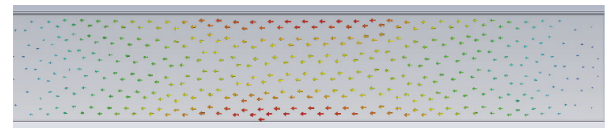

Mode $2\left(J_{2}\right)$

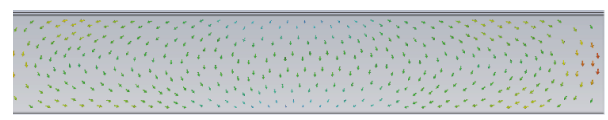

Mode $3\left(J_{3}\right)$

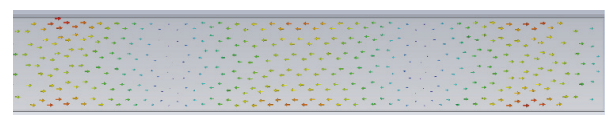

Mode $4\left(J_{4}\right)$

FIgURE 2: Normalized current distribution at $915 \mathrm{MHz}$ of first four associated modes of $160 \times 25 \mathrm{~mm}^{2}$ rectangular plate.

$$
[X][J]_{n}=\lambda_{n}[R][J]_{n}
$$

where $R$ and $X$ are real and imaginary components of MoM impedance matrix; $J_{n}$ are eigencurrents and $\lambda_{n}$ are eigenvalues:

$$
\begin{aligned}
& R=\frac{Z+Z^{*}}{2}, \\
& X=\frac{Z-Z^{*}}{2 j} .
\end{aligned}
$$

Furthermore, because of the orthogonality of characteristics modes, they can be used to derive total surface current $J$ of an antenna as follows:

$$
J=\sum_{n} \frac{V_{n}^{i} J_{n}}{1+\lambda_{n}}
$$

where $V_{n}^{i}$ is model excitation coefficient and it can be obtained as expressed by

$$
V_{n}^{i}=\oiint_{S} J_{n} \cdot E^{i} d s
$$

The product $V_{n}^{i} J_{n}$ determines the coupling between $n$th mode and excitation. It also provides information about which mode of antenna is excited by feed or incident electric field $\left(E^{i}\right)$.

2.2. Characteristic Mode Analysis for Rectangular Plate. The theory of characteristic modes (TCM) has been employed to calculate characteristic modes of a rectangular plate $(160 \times$ $25 \mathrm{~mm}^{2}$ ) mounted on a FR 4 (2 mm thick, loss less) substrate. The multilayer solver of CST Microwave studio has been used for modal analysis.

Figure 2 shows the normalized current distribution (at $915 \mathrm{MHz}$ ) of first four associated characteristic modes of

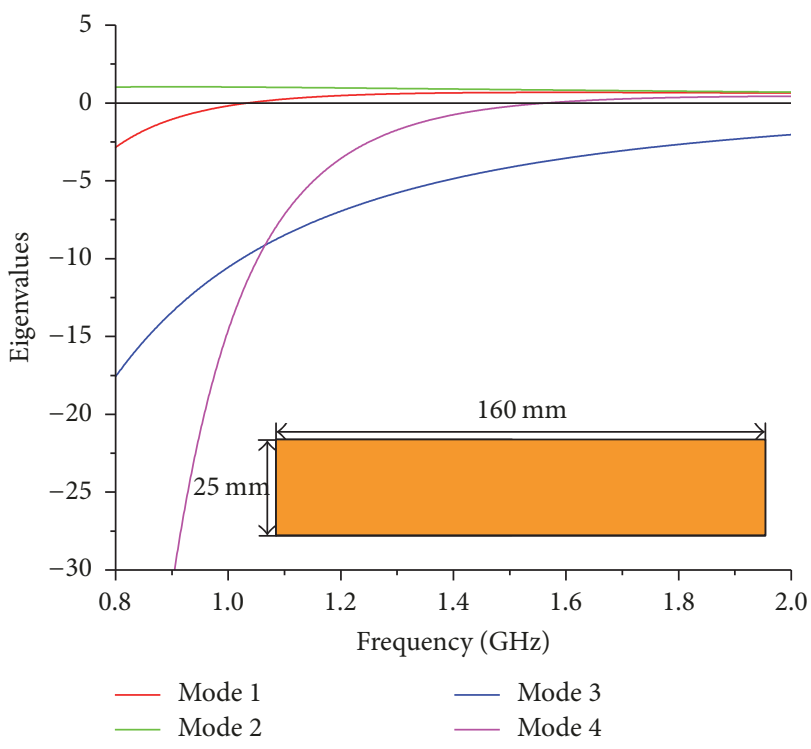

FIGURE 3: Eigenvalues of typical rectangular plate in free space.

rectangular plate (as shown in subset of Figure 3 ). In order to understand behavior of these modes, eigenvalues will be used in combination with normalized current distributions. The eigenvalue associated with resonating modes is zero; it is concluded that the smaller the eigenvalue associated with a mode is, the more efficiently it will contribute to radiation. Moreover, the sign of eigenvalue determines whether a mode is storing electrical energy $\left(\lambda_{n}<0\right)$ or magnetic energy $\left(\lambda_{n}>\right.$ $0)$. The eigenvalues associated with aforementioned modes of rectangular plate in free space are shown in Figure 3. The mode $J_{1}$ resonates near $1.1 \mathrm{GHz}$ as eigenvalue is zero near $1.1 \mathrm{GHz}$. Because of symmetry, the mode $J_{1}$ will propagate even if we use half of the length of rectagular plate. As can be seen from Figure 2, the mode $J_{2}$ behaves like an inductive mode; it can also be confirmed from its associated eigenvalue $\left(\lambda_{n}>0\right)$. Furthermore, mode $J_{3}$ represents capacitator like current distribution with eigenvalue $\left(\lambda_{n}<0\right)$. Mode $J_{4}$ is a resonating mode at $1.6 \mathrm{GHz}$.

Another parameter known as modal significance is also very useful in determining how efficiently a mode resonates. Also, with the application of external excitation source, modal significance determines the contribution of particular mode to total radiation. The modal significance associated with characteristic modes of $160 \times 25 \mathrm{~mm}^{2}$ rectangular plate is shown in Figure 4.

Mode 1 and Mode 4 are resonating modes and will contribute to radiation, when excited by a source, since their modal significance approaches 1 (as shown in Figure 4).

Modal significance can be defined as follows:

$$
\mathrm{MS}_{n}=\frac{1}{\left|1+j \lambda_{n}\right|} .
$$

Additionally, characteristic angle computed from eigenvalues as expressed by (6) is also very useful in determining exact resonance frequencies especially where more than one mode needs to be excited (e.g., circular polarized antenna design). 


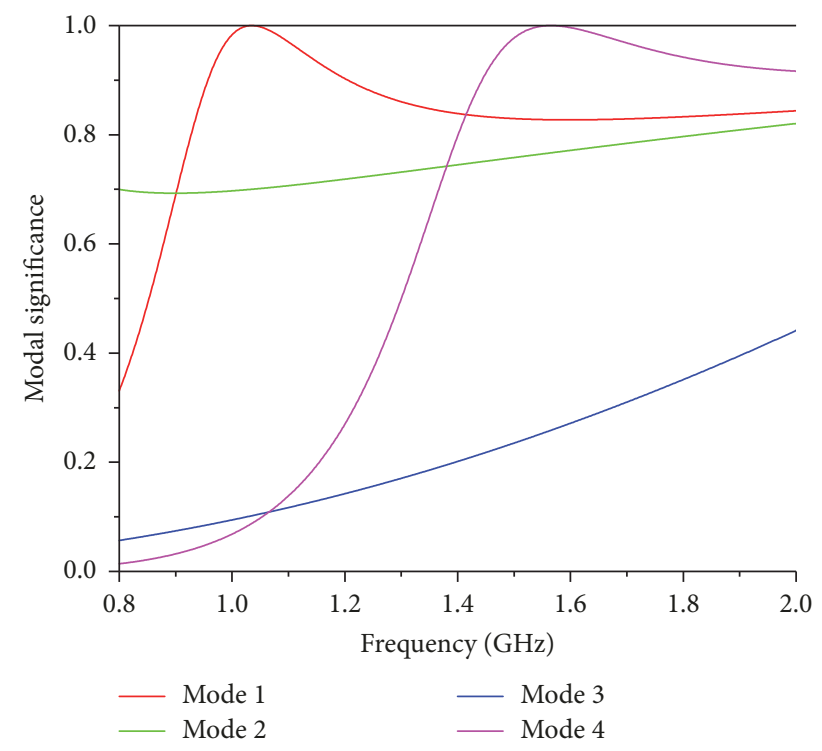

Figure 4: Modal significance of first four modes of $160 \times 25 \mathrm{~mm}^{2}$ rectangular plate.

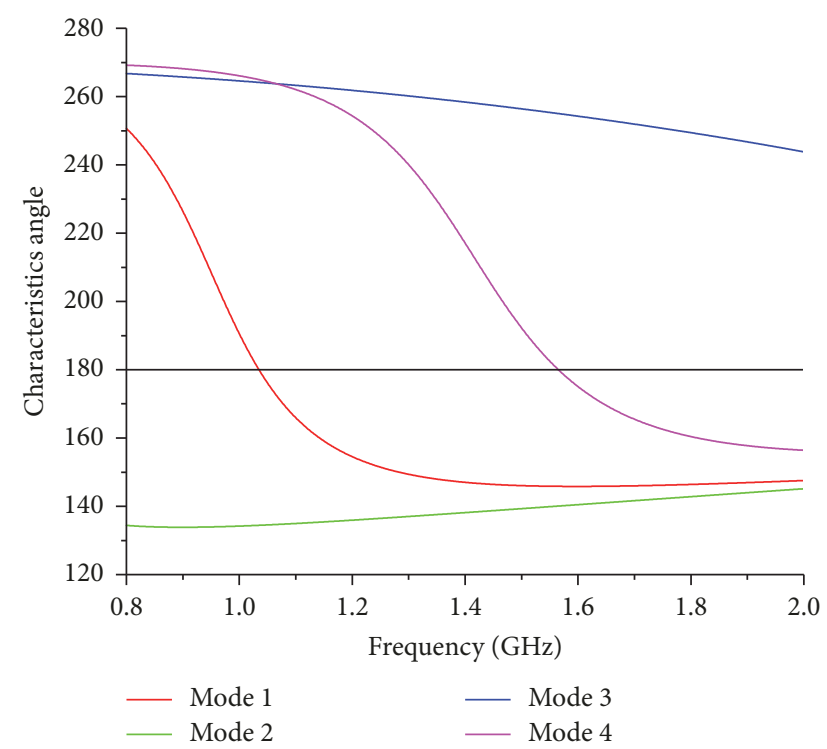

FIgURE 5: Characteristic angle of first four modes of $160 \times 25 \mathrm{~mm}^{2}$ rectangular plate.

The characteristic angle associated with first four modes of $160 \times 25 \mathrm{~mm}^{2}$ rectangular plate are presented in Figure 5. For resonating modes $\alpha_{n}=180^{\circ}$, therefore, Mode 1 and Mode 4 are resonating modes at $1.1 \mathrm{GHz}$ and $1.6 \mathrm{GHz}$, respectively.

$$
\alpha_{n}=180^{\circ}-\tan ^{-1} \lambda_{n}
$$

\section{RFID Tag Antenna Design Using CMA}

3.1. CMA of Dipole Patch. Start from Mode $2\left(J_{2}\right)$ of aforementioned rectangular plate $\left(160 \times 25 \mathrm{~mm}^{2}\right)$. As discussed earlier Mode 2 is an inductive mode. In order to make it as a resonating mode, a small slot (corresponding to adding

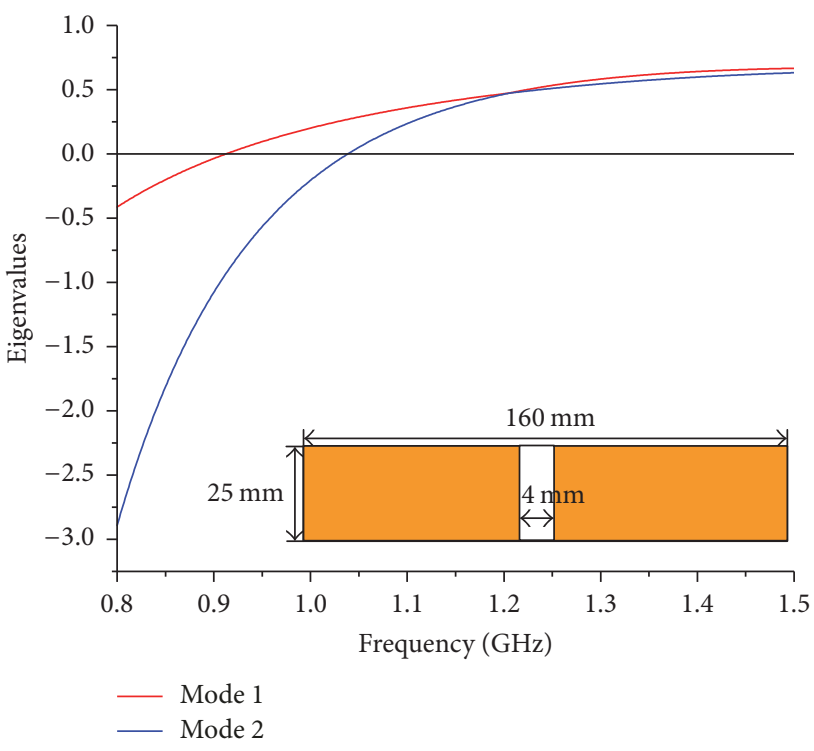

FIGURE 6: Eigenvalues of dipole patch in free space.

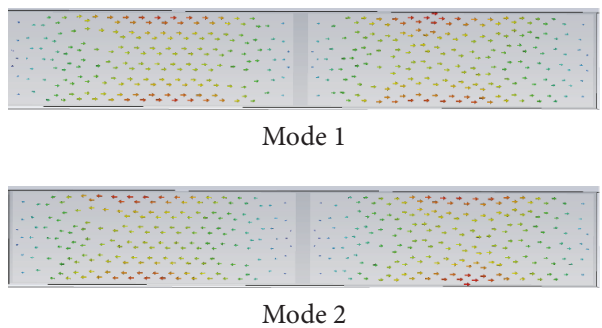

FIGURE 7: Normalized current distribution at $915 \mathrm{MHz}$ of two associated resonating modes of dipole patch.

a capacitance) is created and it becomes dipole like patch as shown in subset of Figure 6. As can be seen from Figure 7, the two modes are resonating at $920 \mathrm{MHz}$ and $1.1 \mathrm{GHz}$, respectively. Furthermore, it can be inferred from normalized current distribution (shown in Figures 2 and 7) that Mode 1 of rectangular plate and Mode 2 of dipole patch are same. It means that the introduction of slot has no effect on this mode.

Inductive Mode 2 of rectangular plate becomes a resonating mode with introduction of capacitance introduced by small slot.

3.2. CMA of Proposed Folded Patch. The dipole patch is working at $920 \mathrm{MHz}$ (US RFID bands $902 \mathrm{MHz}$ to $928 \mathrm{MHz}$ ); however its length is more in order to use it as RFID tag. Due to symmetry, Mode 1 (resonating at $920 \mathrm{MHz}$ ) of dipole patch will still resonate, even if half of the dipole patch is used $(80 \mathrm{~mm})$. In order to reduce the length further the patch is folded with same length on FR 4 substrate as shown in subset of Figure 8. The eigenvalue plot of resulting folded patch antenna is illustrated in Figure 8. To confirm its operation as platform tolerant design, the eigenvalue plot of one resonating mode is depicted in Figure 8, after placing this folded patch in free space and above a finite perfect electric conductor (PEC) boundary. It can be observed from eigenvalue plot 


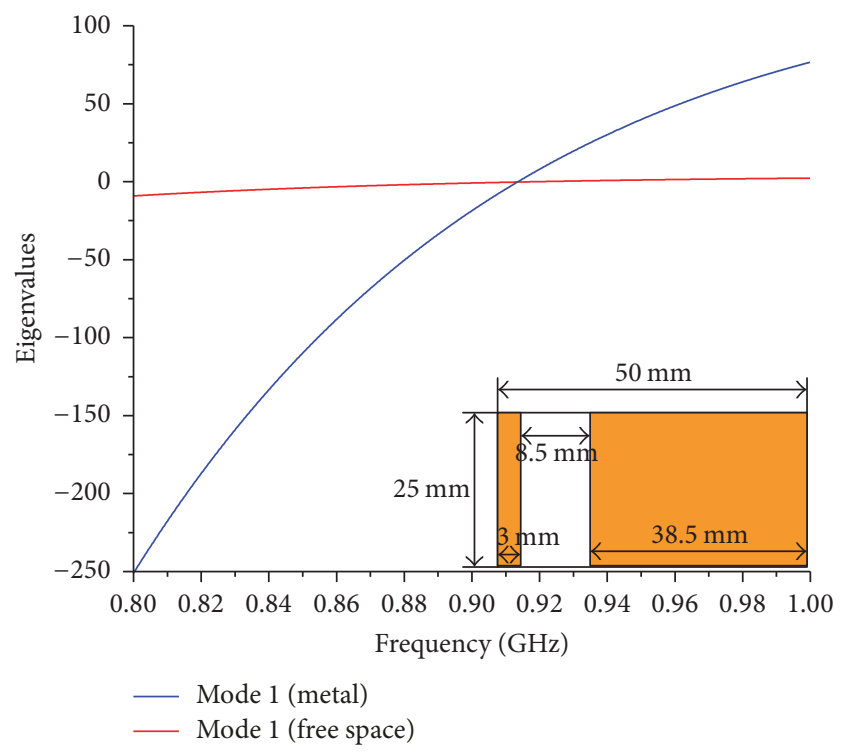

FIGURE 8: Eigenvalues of folded patch in free space and above metal plate.

that the resonance frequency of Mode 1 is almost same for both in free space and above PEC boundary conditions.

However, the slope of eigenvalue plot of Mode 1 is different for two different boundary conditions. Moreover, Mode 1 of folded patch has a steep slope, above metal plate as compared with free space, which means that folded patch has more bandwidth in free space as compared with above metal.

3.3. CMA of Proposed Folded Patch with Tunable Strips. As can be seen from normalized current distribution of folded patch antenna expressed in Figure 9, since the current in Mode 1 is maximum near folded end and decreases towards the slot, it behaves like cavity backed slot antenna. The effective length of current path can be increased by adding two tunable strips on both sides of folded patch near the slot as depicted in subset of Figure 10. Hence, the antenna can be tuned to European RFID band $(866 \mathrm{MHz}-868 \mathrm{MHz})$. The tuning of folded patch to European RFID band can also be confirmed from eigenvalue and modal significance plot (Figure 10) of this folded patch after adding tuning strips.

\section{Simulation Results}

In order to realize an RFID tag antenna, a feeding strategy should be proposed for the folded patch obtained after characteristic mode analysis. Since most of RFID tag chips have capacitive impedance, requiring the tag impedance to be inductive for conjugate match, therefore, inductive coupled loop is a good option to feed this antenna. Besides, the conjugate matching, a separate coupled inductive loop, also provides a versatility of impedance matching with any RFID chip, just by altering loop parameter, without any change in main radiator (folded patch).

The complete antenna geometry of proposed folded patch RFID tag is shown in Figure 11. Finally, the proposed design
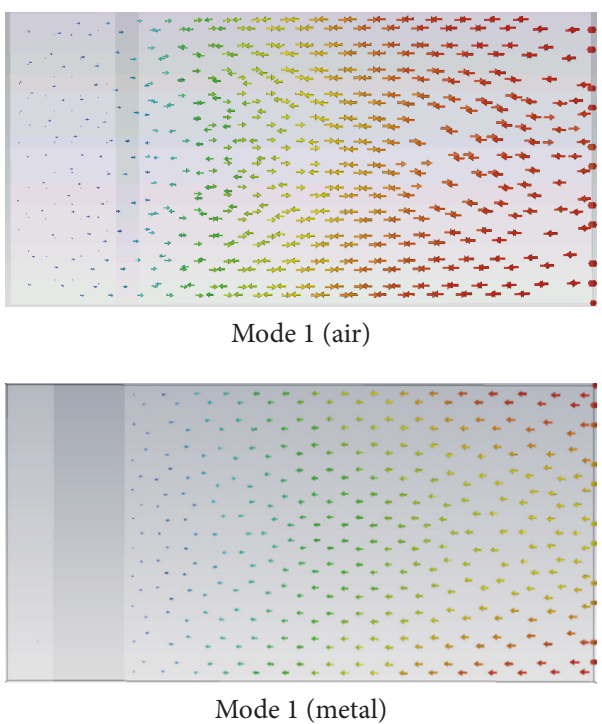

FIgURE 9: Normalized current distribution at $915 \mathrm{MHz}$ of folded patch.

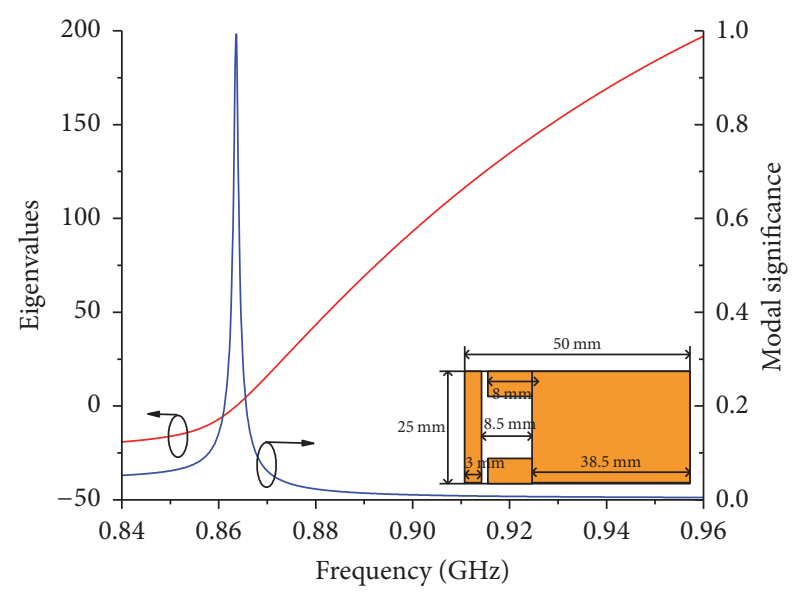

FIGURE 10: Eigenvalue and modal significance of folded patch (with tunable strips) above metal plate.

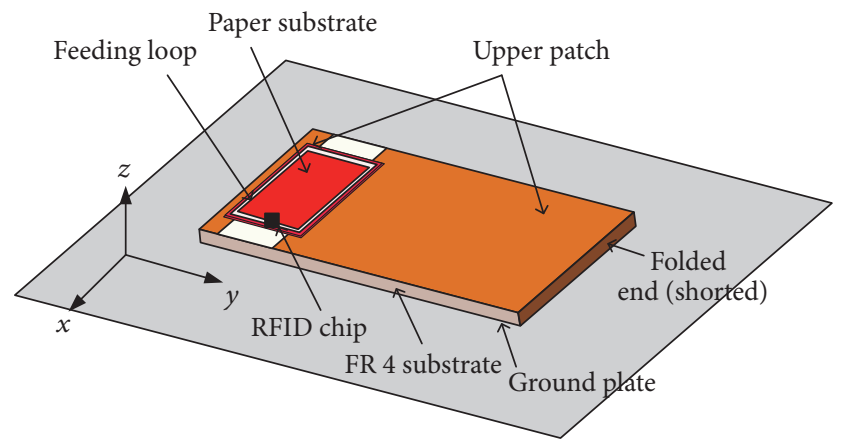

FIGURE 11: Geometry of proposed folded patch RFID tag antenna. 


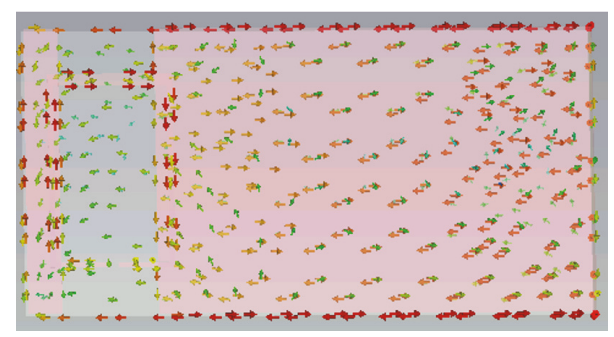

FIGURE 12: Normalized surface current distribution of proposed folded patch RFID tag with feeding loop.

TABLE 1: Parameters of the antenna (mm).

\begin{tabular}{cccccccccc}
\hline$L s$ & $W s$ & $L 1$ & $L 2$ & $L 3$ & $L p$ & $W p$ & $L t$ & $h 1$ & $h 2$ \\
\hline 50 & 25 & 38.55 & 8.5 & 3 & 11 & 17 & 8 & 2 & 0.3
\end{tabular}

consists of a folded patch as main radiator and a small inductive feeding loop in order to match with RFID chip.

Furthermore, Figure 12 shows the surface current distribution after placing the inductive feeding loop above folded patch; it is clear from Figure 12 that the feed loop excites the same mode as expressed in characteristics mode analysis of folded patch antenna (Figure 9). The proposed folded patch is wrapped around a low cost FR 4 substrate $\left(\varepsilon_{r}=4.3, \delta=\right.$ $0.025)$. Furthermore, the inductive feeding loop is printed on a $0.25 \mathrm{~mm}$ thick paper substrate.

The detailed dimensions of proposed design are illustrated in Figure 13. Antenna design parameters involved in simulation are optimized further using frequency domain solver of CST microwave studio and are listed in Table 1. Moreover, Alien Higgs H3 has been used as RFID chip with impedance (30-201 j) computed from equivalent circuit of parallel $1500 \mathrm{ohms}$ resistance and $0.85 \mathrm{pF}$ capacitance.

As shown in Figure 14, the simulated input impedance plot of this tag design confirms the working antenna in American RFID band (US) without tunable strips and can be tuned to European RFID (EU) band with tuning strips. The real and imaginary impedance of antenna is ranging from 30 to $40 \mathrm{Ohms}$ and 180 to $205 \mathrm{Ohms}$, respectively, in required RFID bands, which is best matched with Alien Higgs-3 RFID chip.

To verify antenna performance as platform tolerant design, for simulation purpose, the antenna is placed on two different materials: (1) a $200 \times 200 \mathrm{~mm} 2$ plate with relative permittivity $\varepsilon_{r}=4$, representing a low permittivity dielectric; (2) a $200 \times 200 \mathrm{~mm} 2$ metal plate. For both cases, the power reflection corresponding to conjugate match of this proposed design is shown in Figure 15. The $3 \mathrm{~dB}$ bandwidth of antenna is more than $40 \mathrm{MHz}$ for both cases in US band, with a little bit shift in center frequency for metal case. However, the bandwidth of antenna is enough to protect it from detuning. Moreover, the antenna also provides $3 \mathrm{~dB}$ bandwidth of $30 \mathrm{MHz}$ in EU band. Also, in this case, there is a small shift in center frequency as compared with metal and dielectric case.

Furthermore, the directivity of this tag antenna is $5.87 \mathrm{dBi}$, after mounting it on a $200 \times 200 \mathrm{~mm} 2$ metal plate as depicted in Figure 16.

\section{Measurement and Discussion}

A prototype of proposed RFID tag was fabricated on a ground substrate with one shorted end as shown in Figure 17. Alien Higgs H3 RFID chip is connected with small feeding loop printed on a paper substrate. This paper substrate containing feed loop is then pasted on slot of folded patch.

To validate the performance of antenna on different materials, the input impedance of this tag was measured using Agilent E8363B vector network analyzer by following the procedure expressed in [24].

The impedance measurement setup is depicted in the inset of Figure 18. The outer coats of two coaxial cables were soldered together, while the single end of each cable was attached with open ends of feeding loop. The return loss of antenna was computed by formula given in [24] using parameter measured from this setup.

As compared with simulated results (Figure 18), a better impedance match was observed for 865 to $868 \mathrm{MHz}$ band, whereas the value of measured return loss is more for 902 to $928 \mathrm{MHz}$ band. Overall, the measured results show a good agreement with simulation results with a little reduction in bandwidth. This may be due to some fabrication error or cable loss. Moreover, the measurement procedure also introduces some discrepancies.

Furthermore, the glue used to paste the feeding loop also introduces some change in permittivity and thickness of substrate.

The theoretical read range of RFID tag can be estimated using Friis equation as follows:

$$
R_{\mathrm{tag}}=\frac{\lambda}{4} \sqrt{\frac{P_{r} G_{r} G_{a} \tau}{P_{\mathrm{th}}}}
$$

where $P_{r}, G_{r}$ are transmitted power and gain of RFID reader, $G_{a}$ is gain of tag antenna, and $P_{\mathrm{th}}$ is minimum threshold power of chip.

However, another better and more practical approach to estimate maximum read range with maximum permitted EIRP is to calculate read range first for small value of EIRP with a fixed distance as expressed by

$$
R_{\max }=R_{\text {ref }} \sqrt{\frac{\operatorname{EIRP}_{\text {max }}}{\operatorname{EIRP}_{\text {ref }}}} .
$$

where $R_{\max }$ is maximum read range of the tag, $R_{\text {ref }}$ is reference fixed distance measured in lab, EIRP $_{\max }$ is maximum permitted EIRP $=4$ Watts for most of the regions, and $\mathrm{EIRP}_{\text {ref }}$ is reference EIRP of range measuring equipment. A Tagformance Pro device (from Voyantic Company) based setup was employed to measure maximum read range in lab as illustrated in Figure 19. A frequency sweep is run by the Tagformance with a fixed distance $\left(R_{\text {ref }}\right)$ provided by foam spacer. The theoretical read range is estimated by system software using formula expressed by (8). The measured read range of proposed tag above $200 \times 200 \mathrm{~mm} 2$ metal plate using Tagformance setup is shown in Figure 20. As is clear from Figure 20, the measured read range of proposed tag is $6.2 \mathrm{~m}$ on $200 \times 200 \mathrm{~mm} 2$ metal plate. 


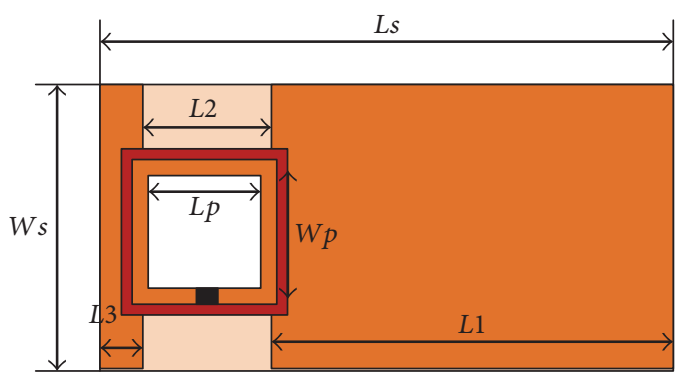

(a)

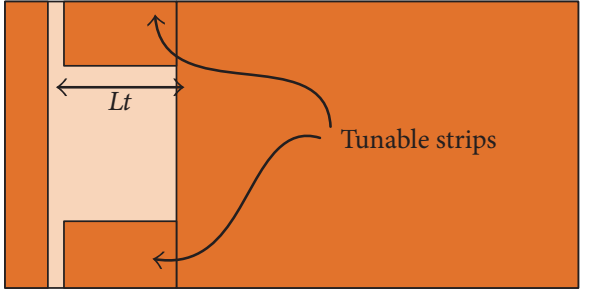

(b)

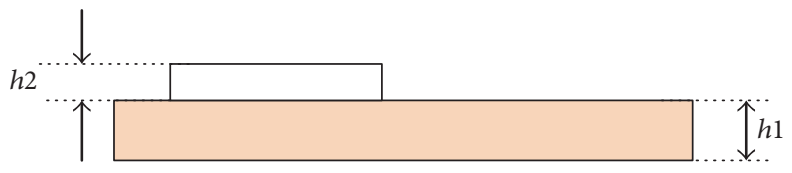

(c)

FIGURE 13: Dimension of proposed folded patch RFID tag. (a) Front view; (b) front view with tuning strips; (c) side view.

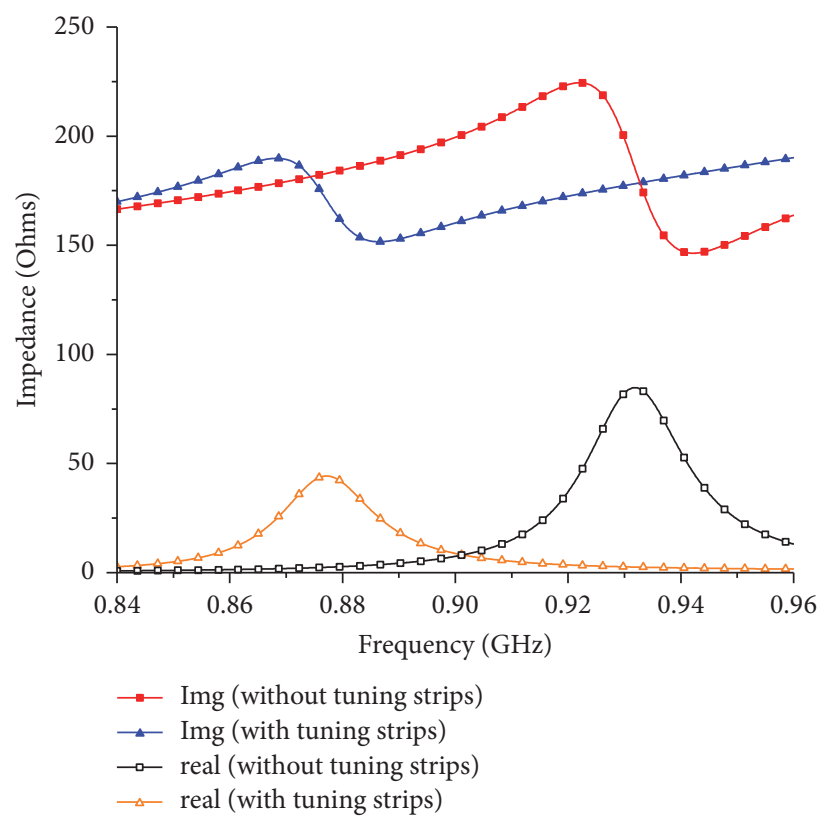

FIGURE 14: Simulated impedance of proposed folded patch RFID tag.

To verify the robustness of this tag solution, the read range of RFID tag was measured after mounting on different materials in outdoor and indoor lab environments with $4 \mathrm{~W}$ EIRP. The measurement setup includes a laptop, Impinj R420 reader, and a circular polarized reader antenna. The read range measurements are carried out at $920 \mathrm{MHz}$.

Furthermore, the different mounting materials used in range measurement were of plastic, wood, glass, and book $\left(200 \times 200 \mathrm{~mm}^{2}\right)$. The maximum measured read range on aforementioned materials is illustrated in Figure 21. Moreover, the maximum read measured on metal plate is $6.5 \mathrm{~m}$, while the measured read range on all other materials is more than $4.5 \mathrm{~m}$ in indoor environment. However, there is a small reduction in read range observed in outdoor environment.

Moreover, the measured read range pattern of this tag design is shown in Figure 22. A good unidirectional read range pattern with maximum read distance of $6.5 \mathrm{~m}$ is acquired in $x z$-plane. Also, it can be observed from Figure 22 that the maximum read angle is tilted since the surface current distribution is more near folded edge.

The comparison of proposed design with some other RFID tags in terms of size and performance is listed in Table 2. 


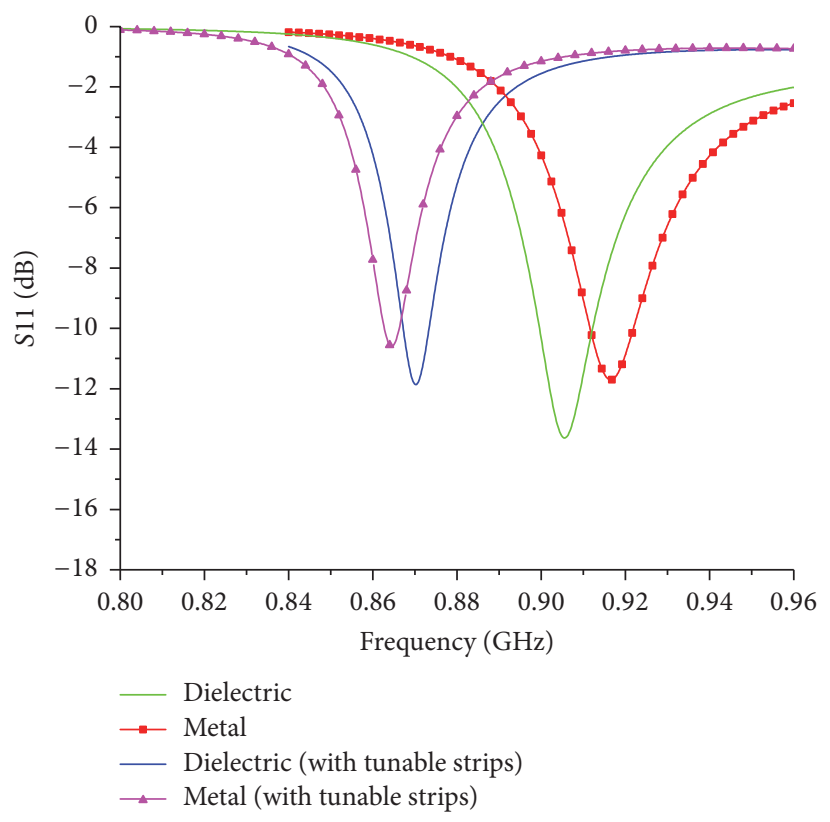

FIGURE 15: Simulated return loss of proposed RFID tag on $200 \times 200 \mathrm{~mm}^{2}$ dielectric and metal plate.

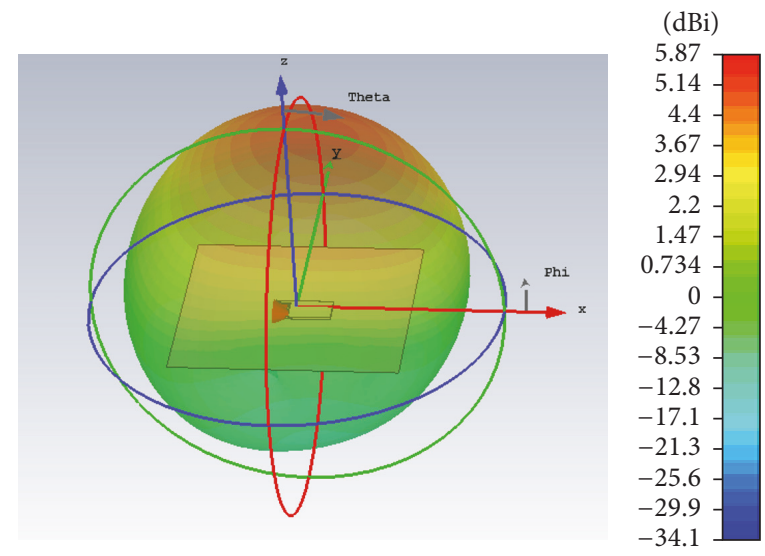

FIGURE 16: Radiation pattern of tag on $200 \times 200 \mathrm{~mm} 2$ metal plate.

TABLE 2: Comparison of proposed antenna with other several designs.

\begin{tabular}{|c|c|c|c|c|}
\hline Reference & Size $\left(\mathrm{mm}^{3}\right)$ & Metal plate size $\left(\mathrm{mm}^{2}\right)$ & $\begin{array}{l}\text { Read range }(\mathrm{m}) \\
\text { (metal/dielectric) }\end{array}$ & Tunable \\
\hline This work & $50 \times 25 \times 2$ & $200 \times 200$ & $6.5 / 4.5$ & Yes \\
\hline$[14]$ & $56 \times 25 \times 3.5$ & $500 \times 500$ & $4 /-$ & No \\
\hline [15] & $55 \times 41.5 \times 3$ & $200 \times 200$ & $6.1 / 14$ & No \\
\hline$[16]$ & $88 \times 60 \times 0.76$ & $150 \times 150$ & 5.4/5.7 (body) & No \\
\hline$[17]$ & $30 \times 30 \times 3$ & $200 \times 200$ & $7 / 3.5$ & No \\
\hline$[18]$ & $84 \times 41 \times 3.2$ & $200 \times 200$ & $5.6 / 8$ & No \\
\hline
\end{tabular}




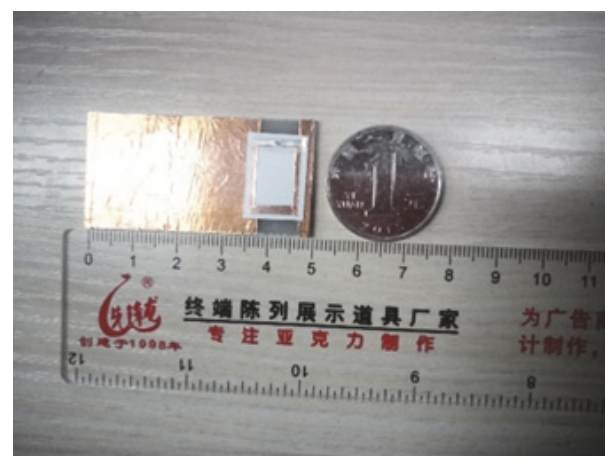

FIGURE 17: Fabricated prototype of proposed RFID tag antenna.

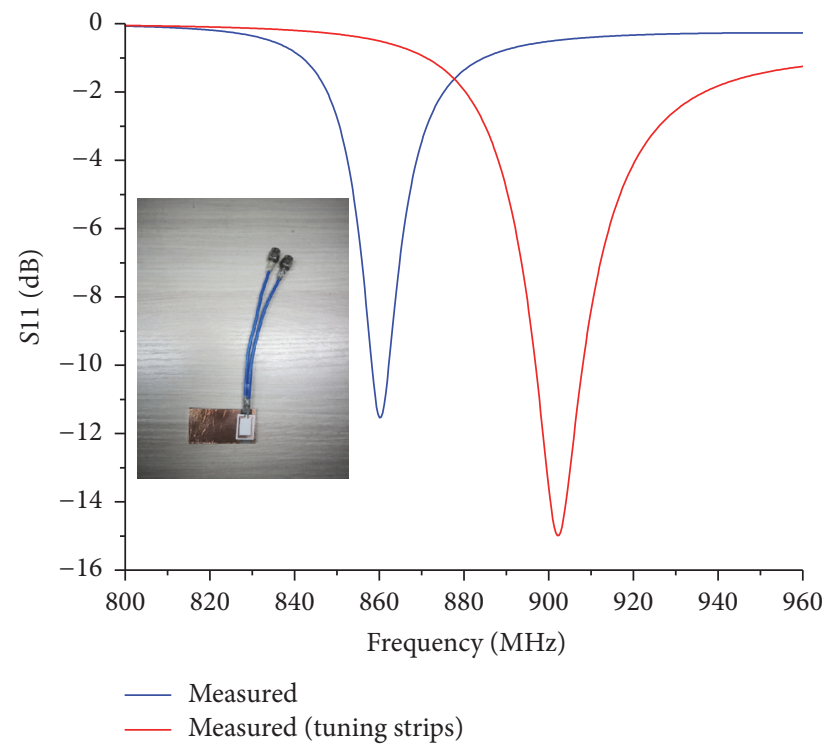

FIGURE 18: Measured return loss of proposed RFID tag on $200 \times$ $200 \mathrm{~mm}^{2}$ metal plate.

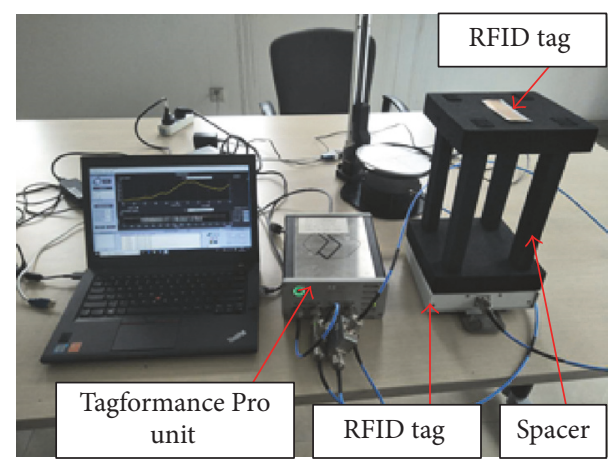

FIGURE 19: Tagformance based read range measuring setup.

\section{Conclusion}

In Internet of Things (IoT), every object can be connected to Internet through a unique digital code to identify it on global scale. RFID in combination with IoT has opened a new paradigm and era of applications. In order to tag a huge number

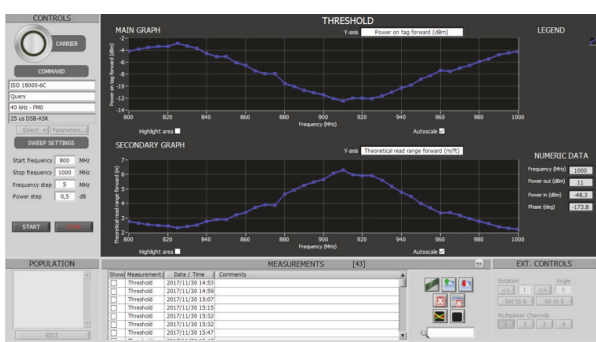

FIGURE 20: Theoretical read range proposed RFID tag on $200 \times$ $200 \mathrm{~mm}^{2}$ metal plate using Tagformance.

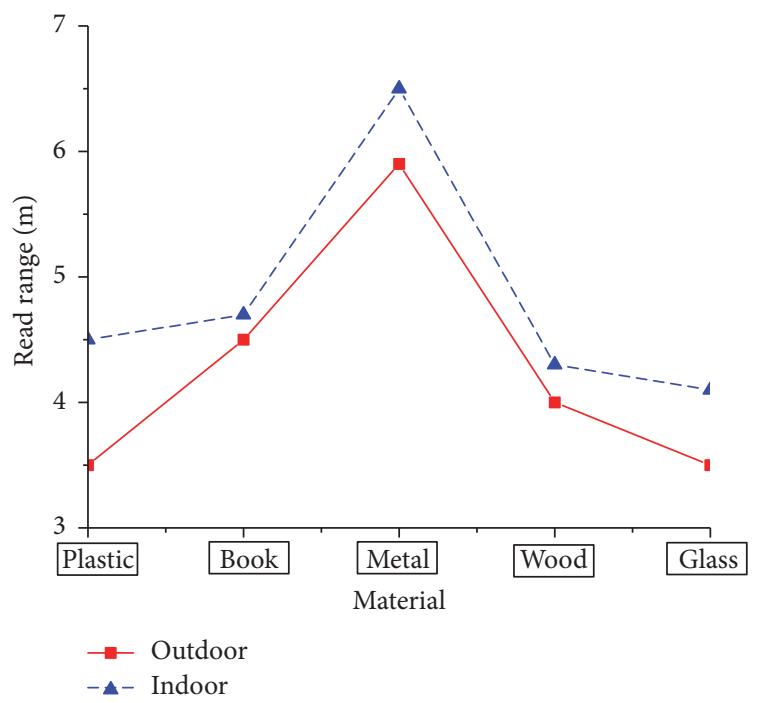

FIGURE 21: Measured read range of proposed RFID tag on block of different materials $\left(200 \times 200 \mathrm{~mm}^{2}\right)$.

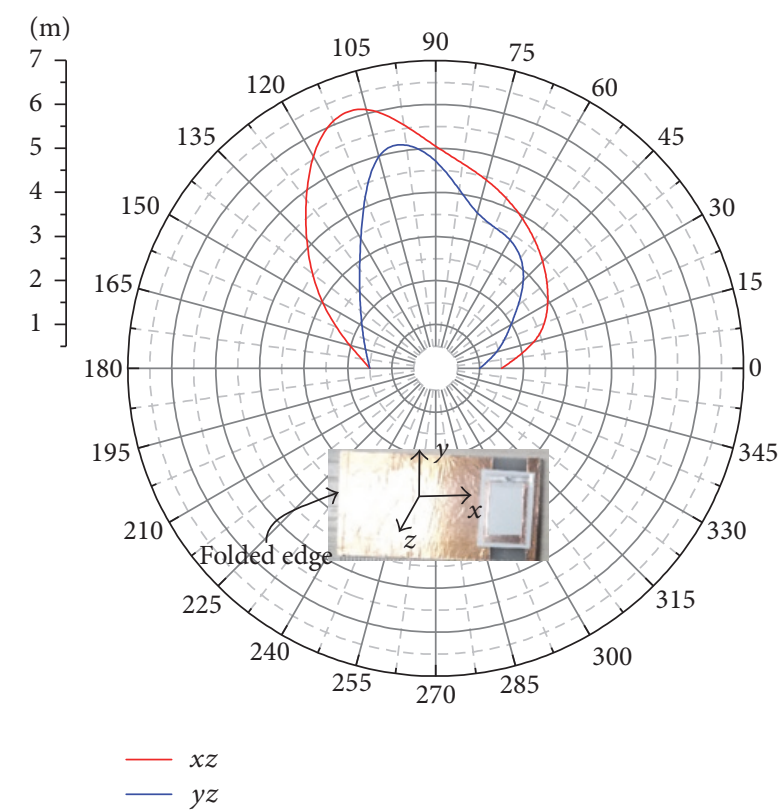

FIGURE 22: Measured read range pattern of proposed RFID tag on $200 \times 200 \mathrm{~mm}^{2}$ metal plate. 
of things, it is cost-effective to use one RFID tag antenna for different things. Therefore, in this paper, a platform tolerant folded patch antenna with tunable capability is proposed for UHF RFID and IoT applications using characteristic mode analysis. The proposed design consists of a folded patch wrapped around FR 4 substrate and a small inductive feeding loop printed on a paper substrate. The proposed folded patch is optimized using CMA to resonate at $915 \mathrm{MHz}$. Since this tag has a separate radiating and feeding element, therefore it can be reusable and has a versatility of impedance matching with any RFID chip. Furthermore, in order to prove platform tolerant capability of proposed RFID tag, its read range is measured after mounting on different material. The maximum read range of RFID tag is $4.5 \mathrm{~m}$ in free space or on dielectrics and $6.5 \mathrm{~m}$ above $200 \times 200 \mathrm{~mm}^{2}$ metal plate, respectively. The platform tolerant capability of this antenna makes it favorable for integration with IoT sensors, which can be used to sense environmental effects and processes. Moreover, the low cost and tunable features of this antenna make it suitable candidate for RFID and IoT applications.

\section{Conflicts of Interest}

The authors declare that there are no conflicts of interest regarding the publication of this paper.

\section{References}

[1] A. Zanella, N. Bui, A. P. Castellani, L. Vangelista, and M. Zorzi, "Internet of things for smart cities," IEEE Internet of Things Journal, vol. 1, no. 1, pp. 22-32, 2014.

[2] D. Pugh, "The Internet of Things About IDTechEx IDTechEx provides clarity on emerging technologies".

[3] S. M. Riazul Islam, D. Kwak, M. Humaun Kabir, M. Hossain, and K.-S. Kwak, "The internet of things for health care: a comprehensive survey," IEEE Access, vol. 3, pp. 678-708, 2015.

[4] L. Catarinucci, D. de Donno, L. Mainetti et al., "An IoT-aware architecture for smart healthcare systems," IEEE Internet of Things Journal, vol. 2, no. 6, pp. 515-526, 2015.

[5] M. Alhussein, "Monitoring Parkinson's Disease in Smart Cities," IEEE Access, vol. 5, pp. 19835-19841, 2017.

[6] G. Marrocco, "RFID \& IoT: a Synergic Pair," IEEE RFID Virtual Journal, vol. 8, pp. 1-21, 2015.

[7] D. M. Dobkin, The RF in RFID.UHF RFID in Practice, Elsevier, Newnes, MA, USA, 2nd edition, 2013.

[8] Y. Huang and G. Li, "Descriptive models for Internet of things," in Proceedings of the 2010 International Conference on Intelligent Control and Information Processing, ICICIP 2010, pp. 483-486, China, August 2010.

[9] T. Yan and Q. Wen, "A secure mobile RFID architecture for the Internet of Things," in Proceedings of the 2010 IEEE International Conference on Information Theory and Information Security, ICITIS 2010, pp. 616-619, China, December 2010.

[10] N. Vikram and K. R. Kashwan, "Design of ISM band RFID reader antenna for IoT applications," in Proceedings of the 2016 IEEE International Conference on Wireless Communications, Signal Processing and Networking, WiSPNET 2016, pp. 1818-1821, India, March 2016.
[11] E. Perret, S. Tedjini, and R. S. Nair, "Design of antennas for UHF RFID tags," Proceedings of the IEEE, vol. 100, no. 7, pp. 23302340, 2012

[12] A. Hamani, M. C. E. Yagoub, T.-P. Vuong, and R. Touhami, "A novel broadband antenna design for UHF RFID tags on metallic surface environments," IEEE Antennas and Wireless Propagation Letters, vol. 16, pp. 91-94, 2017.

[13] H.-D. Chen, C.-Y. Sim, C.-H. Tsai, and C. Kuo, "Compact Circularly Polarized Meandered-Loop Antenna for UHF-Band RFID Tag," IEEE Antennas and Wireless Propagation Letters, vol. 15, pp. 1602-1605, 2016.

[14] J. Zhang and Y. Long, "A dual-layer broadband compact UHF RFID tag antenna for platform tolerant application," IEEE Transactions on Antennas and Propagation, vol. 61, no. 9, pp. 4447-4455, 2013.

[15] H. Li, J. Zhu, and Y. Yu, "Compact Single-Layer RFID Tag Antenna Tolerant to Background Materials," IEEE Access, vol. 5, pp. 21070-21079, 2017.

[16] M. Polivka and M. Svanda, "Stepped impedance coupled-patches tag antenna for platform-tolerant UHF RFID applications," Institute of Electrical and Electronics Engineers. Transactions on Antennas and Propagation, vol. 63, no. 9, pp. 3791-3797, 2015.

[17] F.-L. Bong, E.-H. Lim, and F.-L. Lo, "Flexible Folded-Patch Antenna with Serrated Edges for Metal-Mountable UHF RFID Tag," IEEE Transactions on Antennas and Propagation, vol. 65, no. 2, pp. 873-877, 2017.

[18] A. P. Sohrab, Y. Huang, M. N. Hussein, and P. Carter, "A Hybrid UHF RFID Tag Robust to Host Material," IEEE Journal of Radio Frequency Identification, vol. 1, no. 2, pp. 163-169, 2017.

[19] M. Cabedo-Fabres, E. Antonino-Daviu, A. Valero-Nogueira, and M. F. Bataller, "The theory of characteristic modes revisited: a contribution to the design of antennas for modern applications," IEEE Antennas and Propagation Magazine, vol. 49, no. 5, pp. 52-68, 2007.

[20] E. Antonino-Daviu, M. Cabedo-Fabrés, M. Ferrando-Bataller, and M. Gallo, "Design of a multimode MIMO antenna using the theory of characteristic modes," Radioengineering, vol. 18, no. 4, pp. 425-430, 2009.

[21] E. A. Elghannai and R. G. Rojas, "Modal-based approach to tune and enhance the frequency and dielectric bandwidth of a UHF-RFID tag antenna mounted on a dielectric substrate," in Proceedings of the IEEE Antennas and Propagation Society International Symposium, APS 2015, pp. 161-162, Canada, July 2015.

[22] Z. Liang, J. Ouyang, M. Gao, and X. Cui, "A small RFID tag antenna for metallic object using characteristic mode," in Proceedings of the 2017 IEEE International Symposium on Antennas and Propagation \& USNC/URSI National Radio Science Meeting, pp. 533-534, San Diego, CA, USA, July 2017.

[23] Y. Gao, R. Ma, Q. Zhang, and C. Parini, "Design of very-lowprofile circular UHF small antenna using characteristic mode analysis," IET Microwaves, Antennas \& Propagation, vol. 11, no. 8, pp. 1113-1120, 2017.

[24] X. Qing, C. K. Goh, and Z. N. Chen, "Impedance characterization of rfid tag antennas and application in tag co-design," IEEE Transactions on Microwave Theory and Techniques, vol. 57, no. 5, pp. 1268-1274, 2009. 


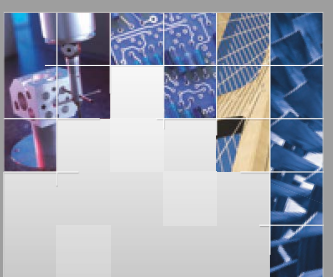

\section{Enfincering}
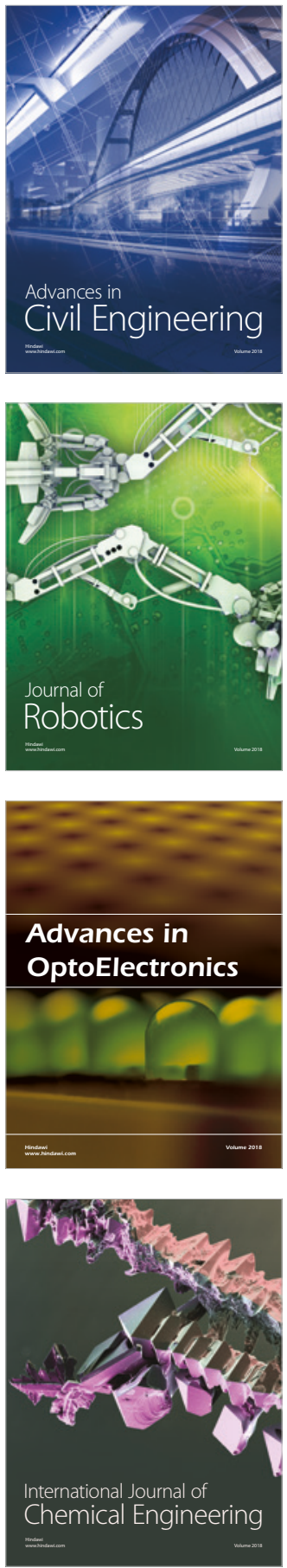

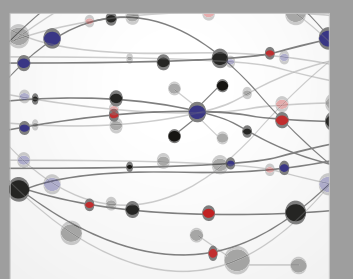

\section{Rotating \\ Machinery}

The Scientific World Journal

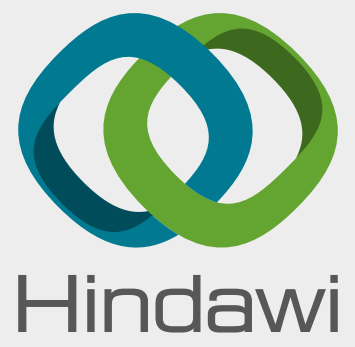

Submit your manuscripts at

www.hindawi.com
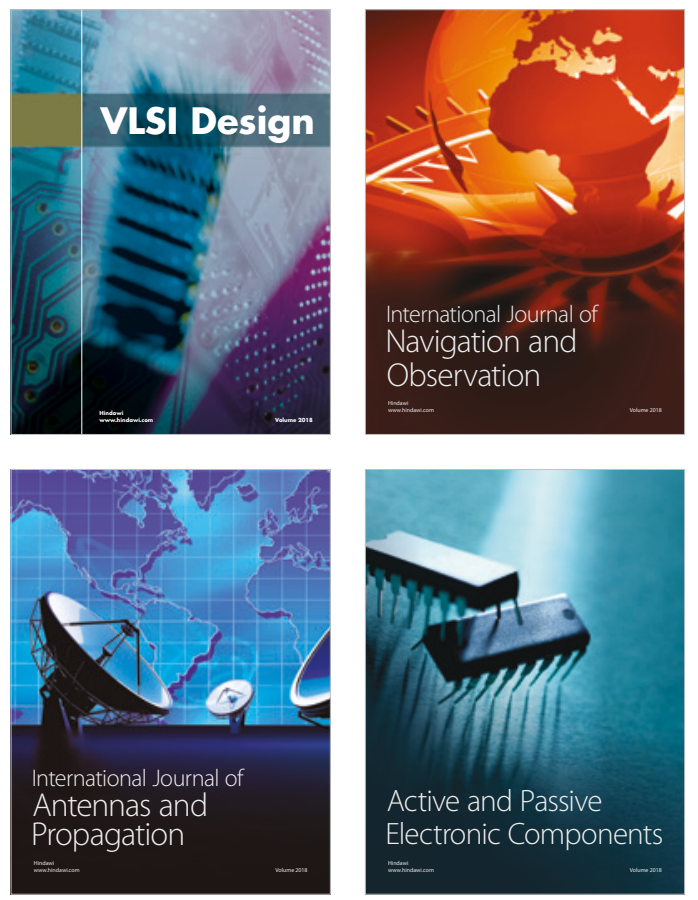
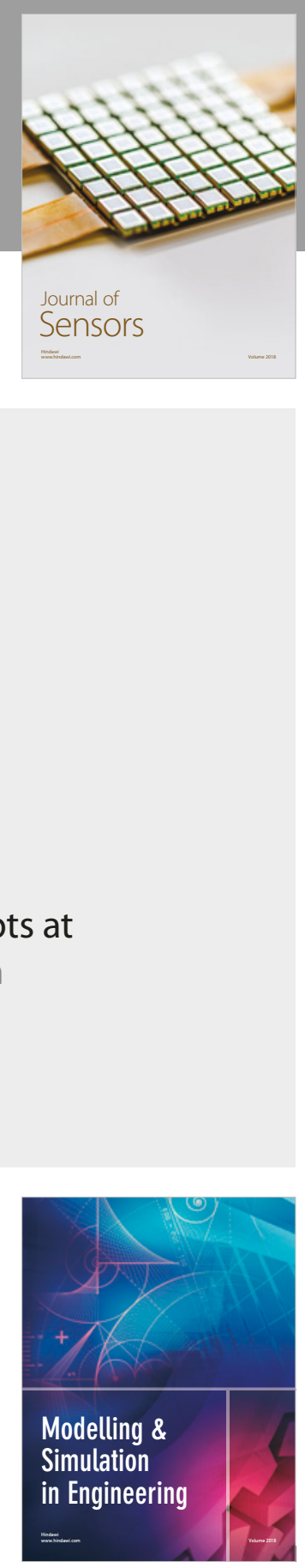

\section{Advances \\ Multimedia}
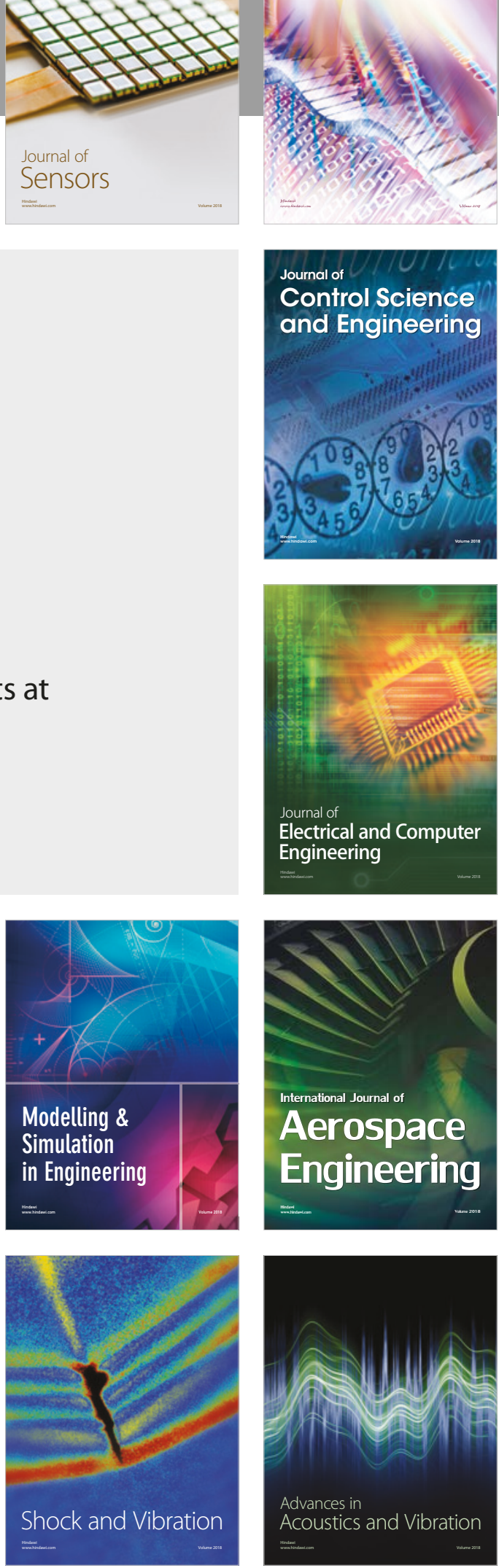